$1-25-2006$

\title{
On the Determination of Residual Stress and Mechanical Properties by Indentation
}

\author{
Xi Chen \\ Columbia University \\ Jin Yan Yan \\ University of Delaware \\ Anette M. Karlsson \\ Cleveland State University, a.karlsson@csuohio.edu
}

Follow this and additional works at: https://engagedscholarship.csuohio.edu/enme_facpub

Part of the Mechanical Engineering Commons

How does access to this work benefit you? Let us know!

\section{Publisher's Statement}

NOTICE: this is the author's version of a work that was accepted for publication in Materials

Science and Engineering A. Changes resulting from the publishing process, such as peer review, editing, corrections, structural formatting, and other quality control mechanisms may not be reflected in this document. Changes may have been made to this work since it was submitted for publication. A definitive version was subsequently published in Materials Science and Engineering A, 416, 1-2, (01-25-2006); 10.1016/j.msea.2005.10.034

\section{Original Citation}

Chen, X., Yan, J., and Karlsson, A. M., 2006, "On the Determination of Residual Stress and Mechanical Properties by Indentation," Materials Science \& Engineering A, 416(1-2) pp. 139-149.

This Article is brought to you for free and open access by the Mechanical Engineering Department at EngagedScholarship@CSU. It has been accepted for inclusion in Mechanical Engineering Faculty Publications by an authorized administrator of EngagedScholarship@CSU. For more information, please contact library.es@csuohio.edu. 


\title{
On the determination of residual stress and mechanical properties by indentation
}

\author{
Xi Chen ${ }^{\mathrm{a}, *}$, Jin Yan ${ }^{\mathrm{b}}$, Anette M. Karlsson ${ }^{\mathrm{b}}$ \\ a Department of Civil Engineering and Engineering Mechanics, Columbia University, New York, NY 10027-6699, USA \\ ${ }^{\mathrm{b}}$ Department of Mechanical Engineering, University of Delaware, Newark, DE 19716-3140, USA
}

\section{Introduction}

The mechanical reliability of bulk materials and coatings (e.g. fatigue, fracture, corrosion and wear) is strongly affected by their residual stress, commonly introduced by thermal mismatch, or mechanical and thermal processing. The most critical residual stress component in coatings is the in-plane equi-biaxial residual stress typically caused by lattice spacing mismatch and thermal expansion mismatch between the film and substrate. Moreover, the mechanical properties and residual stress of all materials are temperature dependent. For thin coatings and substrates, the dependency becomes exacerbated with a change in temperature. This difference in behavior due to temperature change can be critical in service, since mismatch between coating and substrate leads to high residual stress and ultimately coating failure [1]. For example, coatings used for thermal protection in gas turbine engines, experience local compressive stresses as high as several GPa at ambient [2-4]. Thus, in order to achieve a reliable engineering design, the actual level and sign of residual stress in

\footnotetext{
* Corresponding author. Tel.: +1 2128543787 ; fax: +1 2128546267.

E-mail address: xichen@civil.columbia.edu (X. Chen).
}

the specimen must be determined, frequently by measurements, since associated material properties are many times unknown.

There are many challenges associated with measuring properties of small scale structures, but instrumented indentation is widely used to probe mechanical properties such as Young's modulus, $E$, and yield strength, $\sigma_{\mathrm{y}}$, for most engineering materials. Therefore, we will extend the usefulness of this method to measure the residual stress, $\sigma_{\text {res }}$.

\subsection{A brief review of the indentation technique}

A variety of techniques have been developed for measuring mechanical properties from indentation load-displacement data of an elastic-perfectly plastic, stress-free bulk material [5-8], which will be review briefly in this subsection. Instrumented indentation is characterized by a sharp rigid indenter (with a half apex angle $\alpha$ ) penetrating normally into a homogeneous solid where the indentation load, $P$, and displacement, $\delta$, are continuously recorded during one complete cycle of loading and unloading (Fig. 1a and b). To simplify the analysis, the indenter is usually modeled as a rigid cone with $\alpha=70.3^{\circ}$, so that the ratio of cross-sectional area to depth is the same as for a 


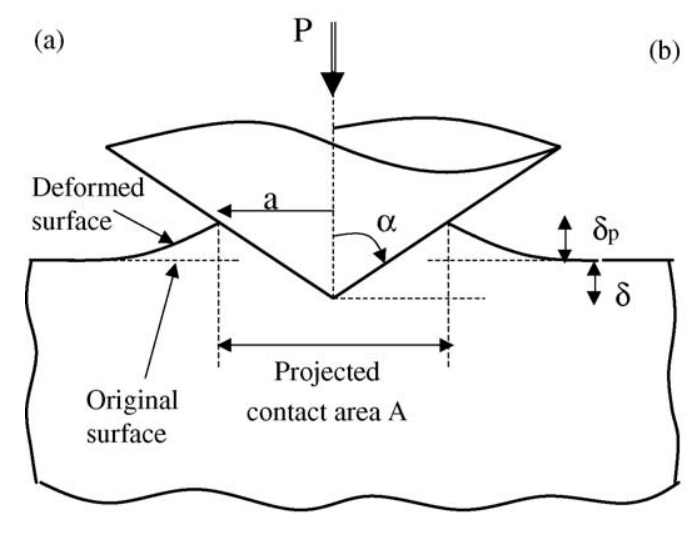

(b)

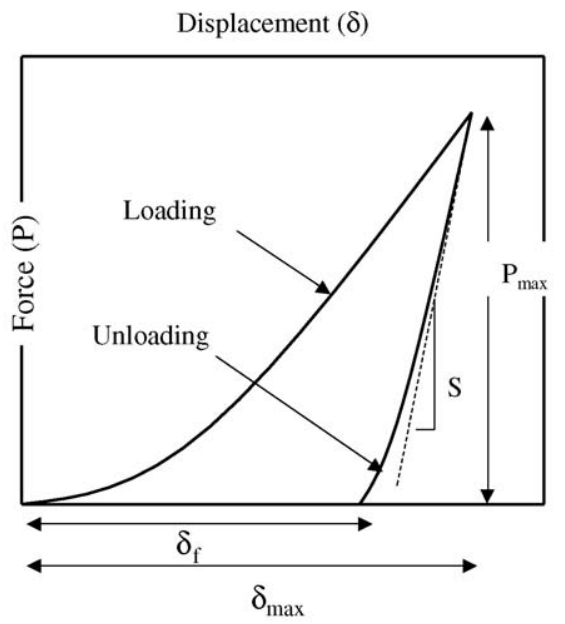

(c)

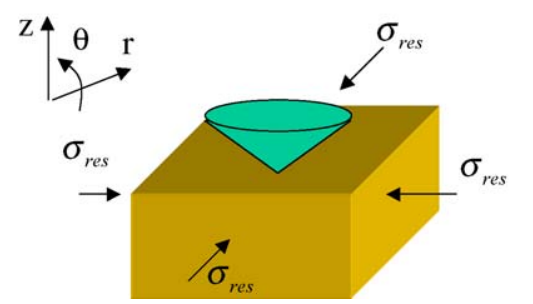

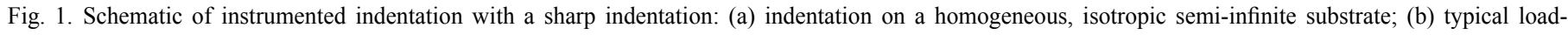
displacement curves obtained from an indentation experiment; (c) conical indentation on a specimen with equi-biaxial in-plane residual stress.

Berkovich or Vickers indenter [9]. By neglecting friction and the finite compliance of the measuring system and the indenter tip, the equations used to extract the hardness $H$ and indentation modulus $M$ are

$H=P / A=c_{\mathrm{b}} \sigma_{\mathrm{y}}$,

and

$S=\gamma \beta \frac{2}{\sqrt{\pi}} M \sqrt{A}$.

Here, the hardness $H$ is defined as the ratio between indentation load $P$, projected contact area $A$ and yield stress $\sigma$. The indentation modulus $M$ is given by the plane-strain modulus, $\bar{E} \equiv E / 1-v^{2}$, for isotropic materials and by a more complicated weighted average of the elastic constants for anisotropic materials [10], where $E$ and $v$ are the Young's modulus and Poisson's ratio of the bulk material, respectively. The contact stiffness $S=\mathrm{d} P / \mathrm{d} \delta$ is obtained from the slope of initial portion of the elastic unloading curve (Fig. 1b). The constant $c_{\mathrm{b}}$ in Eq. (1) is a constraint factor that depends on indenter shape and material properties: $c_{\mathrm{b}}$ increases with $E / \sigma$ and approaches a constant $(\approx 3)$ when $\bar{E} \tan \alpha / \sigma_{\mathrm{y}}>20[9,11]$. $\beta$ is a shape factor, with $\beta=1$ for axisymmetric indenters and $\beta=1.03-1.05$ for indenters with square or rectangular cross-sections [12]. $\gamma=\pi \frac{\pi / 4+0.155 \cot \alpha(1-2 v / 4(1-v))}{(\pi / 2-0.831 \cot \alpha(1-2 v / 4(1-\nu)))^{2}}$ is a correction factor for the conical indenter [13]. Both hardness and stiffness are independent of the indentation depth if the strain gradient effect is ignored $^{1}[14]$.

\footnotetext{
${ }^{1}$ For metals it is observed that the hardness increases with decreasing indentation depth, when the penetration is in the sub-micron regime. This is known as strain gradient plasticity. We ignore such effect by assuming that the indentation depth is sufficiently deep.
}

As the indenter penetrates the specimen, the materials either produce plastic pile-up at the crater rim (when the yield strain, $\sigma_{\mathrm{y}} / E$, is small), or exhibit the elastic sink-in effect (when $\sigma_{\mathrm{y}} / E$ is large) [11]. The amount of pile-up/sink-in is denoted as $\delta_{\mathrm{p}}$ (Fig. 1a). For conical indenters, the projected contact area $A$ is given by

$A=\pi a^{2}=\pi(\tan \alpha)^{2} \delta_{\mathrm{c}}^{2}=24.5 \delta_{\mathrm{c}}^{2}$,

where the contact depth:

$\delta_{\mathrm{c}}=\delta+\delta_{\mathrm{p}}$

Eq. (4) contains contributions of both plastic pile-up around the indenter and elastic sink-in, which is counted negative. It is obvious from Eqs. (3) and (4), that the projected contact area depends on $\delta_{\mathrm{p}}$. Oliver and Pharr [5] proposed an elastic model for determining the contact area in which plastic pile-up is neglected:

$\left|\delta_{\mathrm{p}}\right|=\frac{\varepsilon P_{\max }}{S_{\max }}$

where $\varepsilon=0.75$ for a conical indenter. Alternatively, the pile-up and contact area can be measured experimentally or determined from the numerical analysis (such as the finite element method or molecular dynamics). Once $A$ is determined properly, the hardness is then obtained from Eq. (1) and the stiffness can be derived from Eq. (2), which allows one to measure $\bar{E}$ and $\sigma_{\mathrm{y}}$ by using the indentation technique.

\subsection{The effect of in-plane residual stress}

Several attempts have been made to incorporate residual stress measurements from instrumented indentation. Tsui et al. 1996 [15] used standard nanoindentation techniques to investigate the influence of uniaxial and biaxial in-plane stresses 
(applied to the specimen by bending) on the hardness and elastic modulus measurements of aluminum. The projected contact areas were measured by optical techniques. It was found that the hardness and elastic modulus thus calculated were essentially independent of the residual stress level, which was verified by a parallel finite element simulation [16]. Based on the assumption that the hardness is unaffected by tensile or compressive elastic residual stress, several general methods was proposed for determining the in-plane equi-biaxial residual stress by conical or spherical indentation. Most approaches compare the contact depth (or contact area, or load-displacement curve) of the stressed and unstressed specimens, from which $\sigma_{\text {res }}$ can be estimated [17-20]. In some other studies [21,22], the residual compression was seen to enhance the pile-up and residual tension reduced $\delta_{\mathrm{p}}$. The ratio between the projected and nominal contact areas, i.e. $\left(\delta_{\mathrm{c}} / \delta\right)^{2}$, was calculated as a function of $\sigma_{\text {res }}$ by using finite element analysis. Therefore, the authors suggests, the in-plane residual stress can be obtained if $\delta_{\mathrm{p}}$ can be measured properly for a given material [21,22].

The above techniques have several disadvantages: they either require testing on a stress-free specimen as a reference, or determining the contact depth accurately, which is often not feasible. In addition, only few materials were tested which may lead to incomplete conclusions, ${ }^{2}$ and a complete examination of all possible combinations of material properties and residual stresses remains yet to be developed.

Can we measure all three unknowns, $\left(E, \sigma_{\mathrm{y}}, \sigma_{\text {res }}\right)$ from one simple indentation test? How do the different residual stress and mechanical property combinations affect the pile-up, projected contact area, plastic zone, and other indentation mechanisms? The finite element method is useful for this purpose because it provides a convenient way of measuring the projected contact area between indenter and material needed for calculation of hardness and stiffness. It is also straightforward to vary materials properties and residual stress over a wide range, such that our numerical analysis would cover most engineering materials used in practice. Thus, in this study, the finite element method (FEM) is used to investigate the effect of residual stress on elastic-plastic indentation of bulk materials and thick coatings.

For indentation on a coating/substrate system, recent study by Chen and Vlassak [11] has shown that the substrate effect is negligible when the coating is softer than the substrate and the indentation depth is less than half of the coating thickness. To simply the present study, we assume there is no substrate effect. That is, for soft coating deposited on a hard substrate, the indentation depth $\delta$ is smaller than $50 \%$ of the coating thickness; and for hard coating on a soft substrate, the coating is very thick compare with $\delta$ (e.g. thermal barrier coatings). This assumption allows us to only focus on the mechanics of indentation on a homogeneous, isotropic bulk material with equi-biaxial in-plane residual stress. The findings of this study can be readily applied to thin film/coatings when the above criterions for neglecting the substrate effect are met.

\footnotetext{
${ }^{2}$ In fact, it will be show later in this study that hardness becomes very sensitive to residual stress when $\sigma_{\mathrm{y}} / E$ gets large.
}

\section{Model definition}

\subsection{Dimensional analysis}

The axisymmetric model for indentation on a bulk material or thick coating with in-plane residual stress is shown in Fig. 1a and c. The substrate is taken to be infinitely deep. The half apex angle of the rigid conical indenter is $\alpha=70.3^{\circ}$. The coating is elastic-perfectly plastic, applicable to most high-strength alloys and ceramic coatings (the effect of work hardening will be explored elsewhere). Recent work by Mesarovic and Fleck [23] has shown that the Poisson's ratio is a minor factor for static indentation. Dimensional analysis dictates the following relationship for hardness and stiffness:

$\frac{P}{\pi a^{2} \sigma_{\mathrm{y}}}=f\left[\frac{\sigma_{\mathrm{res}}}{\sigma_{\mathrm{y}}}, \frac{\sigma_{\mathrm{y}}}{E}\right]$,

$\frac{S}{2 a E}=g\left[\frac{\sigma_{\text {res }}}{\sigma_{\mathrm{y}}}, \frac{\sigma_{\mathrm{y}}}{E}\right]$.

As discussed above, for conical indentation in absent of residual stress, $S / 2 a \bar{E}=\gamma \beta \approx 1.1$ is a constant; and $P / \pi a^{2} \sigma_{\mathrm{y}}=c_{\mathrm{b}}$, which varies with $\sigma_{\mathrm{y}} / E$. Inspired by the fact that the pile-up (or contact area) is significantly affected by the residual stress (discussed below), the third independent equation governing the three unknown variables is

$\frac{\delta_{\mathrm{p}}}{\delta}=H\left[\frac{\sigma_{\mathrm{res}}}{\sigma_{\mathrm{y}}}, \frac{\sigma_{\mathrm{y}}}{E}\right]$.

It should be noted that although $\delta_{\mathrm{p}} / \delta$ is very sensitive to the variations of $\sigma_{\mathrm{y}} / E$ and $\sigma_{\text {res }} / \sigma_{\mathrm{y}}$, it is very difficult to measure the pile-up or sink-in amount during the experiment. We note that the contact radius can be represented by

$a=\left(\delta_{\mathrm{p}}+\delta\right) \tan \alpha=\delta \tan \alpha\left(1+H\left[\frac{\sigma_{\mathrm{res}}}{\sigma_{\mathrm{y}}}, \frac{\sigma_{\mathrm{y}}}{E}\right]\right)$.

Thus, the work done by indentation is

$$
\begin{aligned}
\int_{0}^{\delta_{\max }} P d \delta= & \int_{0}^{\delta_{\max }} \pi a^{2} \sigma_{\mathrm{y}} f\left[\frac{\sigma_{\text {res }}}{\sigma_{\mathrm{y}}}, \frac{\sigma_{\mathrm{y}}}{E}\right] d \delta \\
= & \frac{\pi \tan ^{2} \alpha}{3} f\left[\frac{\sigma_{\text {res }}}{\sigma_{\mathrm{y}}}, \frac{\sigma_{\mathrm{y}}}{E}\right] \\
& \times\left(1+H\left[\frac{\sigma_{\mathrm{res}}}{\sigma_{\mathrm{y}}}, \frac{\sigma_{\mathrm{y}}}{E}\right]\right)^{2} \sigma_{\mathrm{y}} \delta_{\max }^{3} .
\end{aligned}
$$

It is therefore convenient to define a normalized indentation work, which implicitly embeds the variation of pile-up:

$$
\begin{aligned}
\frac{\int_{0}^{\delta_{\max }} P d \delta}{\sigma_{\mathrm{y}} \delta_{\max }^{3}}= & h\left[\frac{\sigma_{\text {res }}}{\sigma_{\mathrm{y}}}, \frac{\sigma_{\mathrm{y}}}{E}\right] \equiv \frac{\pi \tan ^{2} \alpha}{3} f\left[\frac{\sigma_{\text {res }}}{\sigma_{\mathrm{y}}}, \frac{\sigma_{\mathrm{y}}}{E}\right] \\
& \times\left(1+H\left[\frac{\sigma_{\text {res }}}{\sigma_{\mathrm{y}}}, \frac{\sigma_{\mathrm{y}}}{E}\right]\right)^{2} .
\end{aligned}
$$

By varying $\sigma_{\text {res }} / \sigma_{\mathrm{y}}$ and $\sigma_{\mathrm{y}} / E$ in a wide range, the functional forms of $f, g$, and $h$ can be determined from extensive finite element analysis. The hardness $P /\left(\pi a^{2}\right)$, contact stiffness $S$, and 
indentation work ${ }_{0}^{\delta_{\max }} P d \delta$ can be readily obtained from an experimental indentation load-displacement curve (c.f. Fig. 1b), as long as the contact radius a (or the projected contact area A) can be measured properly by take into account the pile-up or sink-in effect. ${ }^{3}$ Finally, the mechanical properties $\left(E, \sigma_{\mathrm{y}}\right)$ and in-plane residual stress $\left(\sigma_{\text {res }}\right)$ of the specimen can then be solved from the reverse analysis:

$$
\begin{aligned}
& \frac{P}{\pi a^{2}}=\sigma_{\mathrm{y}} f\left[\frac{\sigma_{\mathrm{res}}}{\sigma_{\mathrm{y}}}, \frac{\sigma_{\mathrm{y}}}{E}\right], \\
& \frac{S}{2 a}=\bar{E} g\left[\frac{\sigma_{\mathrm{res}}}{\sigma_{\mathrm{y}}}, \frac{\sigma_{\mathrm{y}}}{E}\right], \\
& \delta_{\max } P d \delta \\
& \frac{0}{\delta_{\max }^{3}}=\sigma_{\mathrm{y}} h\left[\frac{\sigma_{\mathrm{res}}}{\sigma_{\mathrm{y}}}, \frac{\sigma_{\mathrm{y}}}{E}\right] .
\end{aligned}
$$

\subsection{Finite element model}

Finite element calculations were performed using the commercial code ABAQUS [24] on Dell workstations. The rigid contact surface option was used to simulate the rigid indenter, and the option for finite deformation and strain was employed. A typical mesh for the axisymmetric indentation model comprises more than 5000 8-node elements. As already mentioned, the substrate material is taken to be elastic-perfectly plastic, with a Von Mises surface to specify yielding. The Coulomb's friction law is used between contact surfaces, and the friction coefficient is taken to be 0.1 , which is also a minor factor for nanoindentation [23]. The equi-biaxial in-plane residual stress is applied to the specimen by means of thermal expansion, followed by conical indentation on the free surface. The projected contact area is calculated directly from the numerical results by analyzing the nodes in contact with the indenter. All three parameters $\left(E, \sigma_{\mathrm{y}}\right.$, $\left.\sigma_{\text {res }}\right)$ are varied over a large range, such that the yield strain $\sigma_{\mathrm{y}} / E$ is varied between 0.001 to 0.1 , and the residual stress $\sigma_{\text {res }} / \sigma_{\mathrm{y}}$ spans from -1 to 1 . Such a wide range covers almost all possible combinations of mechanical properties and residual stress encountered in engineering materials. In addition to calculating the functional forms $f, g$, and $h$, the effects of residual stress on indentation residual stress, plastic zone, and surface profile (i.e. pile-up) are also examined by FEM and elaborated below.

\section{Numerical results}

\subsection{The effect of residual stress on indentation stress}

The stress and strain fields caused by the indentation interact strongly with the pre-existing fields, which in turn affect the hardness, stiffness, and indentation work, discussed in Section 3.3. The effect of in-plane residual (or applied) stress on hardness can be explicated from the view of shear plasticity. Since

\footnotetext{
3 Optical measurements or AFM surface scan are typically used to determine the pile-up (or sink-in) and true projected contact area. If the material has a high yield strain or if the indentation is nearly elastic, then the Oliver-Pharr method (Eq. (5)) can be applied to obtain $\delta_{\mathrm{p}}$ and $a$ accurately.
}

the indentation pressure is compressive and perpendicular to the applied surface, the existence of tensile surface stress in the specimen increases the magnitude of the shear stresses beneath the indenter compared with an unstressed specimen. Consequently, the plastic deformation in the specimen is enhanced, which leads to a reduction of contact hardness $\mathrm{H}$ and normalized pile-up $\delta_{\mathrm{p}} / \delta$ when in-plane residual tensile stress presents.

Some additional insight to the mechanics behind this can be gained by studying the in-plane stress component, $\sigma_{\mathrm{rr}}$, under maximum indentation load for various residual stress, $\sigma_{\text {res }} / \sigma_{\mathrm{y}}$, Fig. 2. When the residual stress is tensile, $\sigma_{\text {res }} / \sigma_{\mathrm{y}}=0.8$, Fig. $2 \mathrm{a}$, the region of indentation compressive stress is smaller than for the initial stress-free case, $\sigma_{\text {res }} / \sigma_{\mathrm{y}}=0$, Fig. $2 \mathrm{~b}$. This gives rise to an apparent lower hardness material. The opposite situation holds for specimen with residual compression (Fig. 2c, $\left.\sigma_{\text {res }} / \sigma_{\mathrm{y}}=-0.8\right)$ : the maximum compressive stress is larger than that in the unstressed sample, and the resulting overall compression zone is much larger.

Additional insight to the change in apparent hardness can be gained by studying the size of the plastic zone at maximum indentation load for various combinations of $E / \sigma$ and $\sigma_{\text {res }} / \sigma_{\mathrm{y}}$, Fig. 3. For $E / \sigma_{\mathrm{y}}=100$ (Fig. 3b), the plastic strain is enlarged with increasing level of residual stress. Since the plastic region is larger and the deformation beneath the indenter is finite plastic without work hardening, when in-plane residual tensile stress presents, the surface is allowed to "sink-in" during the indentation, which leads to the reduction of contact hardness (discussed below). On the other hand, the residual compressive stress results in a smaller plastic region, and with a larger local accumulation. This results in a significant pile-up during indentation (Fig. 4), more resistance to plastic deformation during penetration, and an increase of normalized hardness (see Section 3.3). For hard materials (Fig. 3a with $E / \sigma_{\mathrm{y}}=10$ ) the plastic region is relatively small, and a significant part of the deformation is elastic. Nevertheless, the trend that the plastic zone enlarges with increasing tensile residual stress $\sigma_{\text {res }} / \sigma_{\mathrm{y}}$ is obvious. A softer specimen with $E / \sigma_{\mathrm{y}}=1000$ shows a more significant plastic region (Fig. 3c). For compressive residual stresses the plastic zone is more confined near the impression crater, where a significant pile-up is observed. When the in-plane residual stress is tensile, the plastic strain is distributed more evenly, resulting in a smaller $\delta_{\mathrm{p}} / \delta$. The details of pile-up are presented in the next section.

\subsection{The effect of residual stress on surface profile}

A common output from indentation testing is the characterization of the deformed surface, commonly used to calculated material properties. However, this may not be a reliable parameter when residual stresses are present. For hard materials $\left(E / \sigma_{\mathrm{y}}=10\right)$, a significant amount of the deformation is elastic, and thus only sink-in presents at the indentation crater (Fig. 4a). In addition, for hard materials the surface profile at maximum penetration is essentially independent of the residual stress, $\sigma_{\text {res }} / \sigma_{\mathrm{y}}$. However, upon unloading the deformation mechanism is dominated by a significant elastic recovery that strongly depends on the residual stress level. A smaller but still 


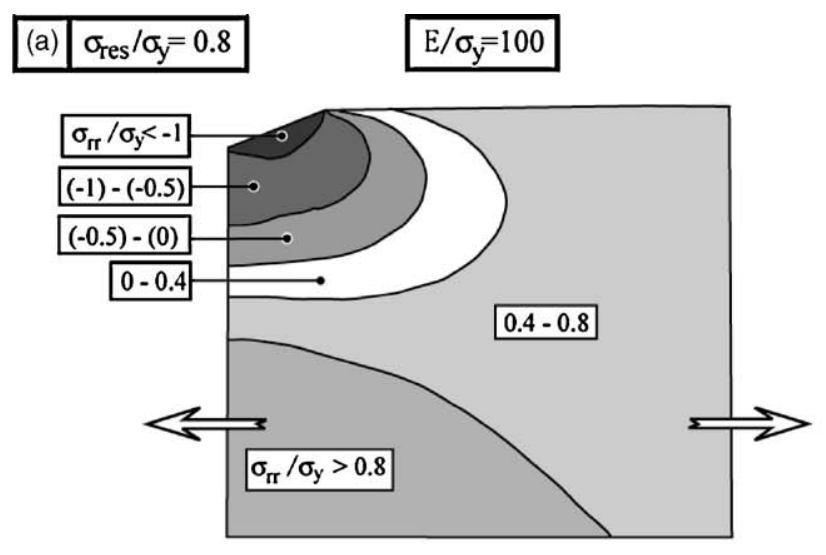

\section{\begin{tabular}{|l|l|}
\hline (b) & $\sigma_{\text {res }} / \sigma_{\mathrm{y}}=0$ \\
\hline
\end{tabular}}

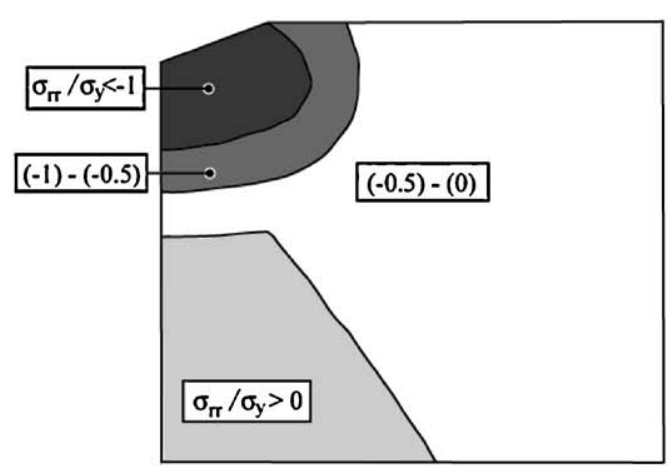

\begin{tabular}{|l|l|}
\hline (c) & $\sigma_{\mathrm{res}} / \sigma_{\mathrm{y}}=-0.8$ \\
\hline
\end{tabular}

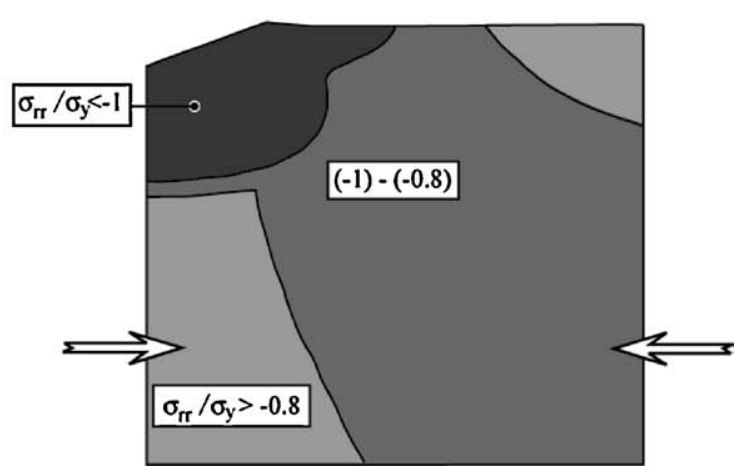

Fig. 2. Contour plots of indentation radial stress $\left(\sigma_{\mathrm{rr}} / \sigma_{\mathrm{y}}\right)$ with different residual stress level, all with $E / \sigma_{\mathrm{y}}=100$ : (a) residual tension $\left(\sigma_{\mathrm{res}} / \sigma_{\mathrm{y}}=0.8\right)$; (b) no residual stress $\left(\sigma_{\text {res }} / \sigma_{\mathrm{y}}=0\right)$; (c) residual compression $\left(\sigma_{\text {res }} / \sigma_{\mathrm{y}}=-0.8\right)$.

noticeable recovery is also observed for $E / \sigma_{\mathrm{y}}=100$ (Fig. 4b). For many high-strength alloys and coatings which have $E / \sigma_{\mathrm{y}} \sim 100$, the effect of residual stress on pile-up/sink-in is obvious through Fig. $4 \mathrm{~b}$ : comparing with the base case where $\sigma_{\text {res }} / \sigma_{\mathrm{y}}=0$, the presence of residual compression significantly enhances the pileup, while residual tension leads to larger plastic zone and more sink-in effect. When the specimen is even softer $\left(E / \sigma_{\mathrm{y}}=1000\right.$, Fig. 4c), indentation only develops pile-up around the crater rim and the amount of pile-up increases with increasing residual compression. The deformation is finite plastic and the plastic

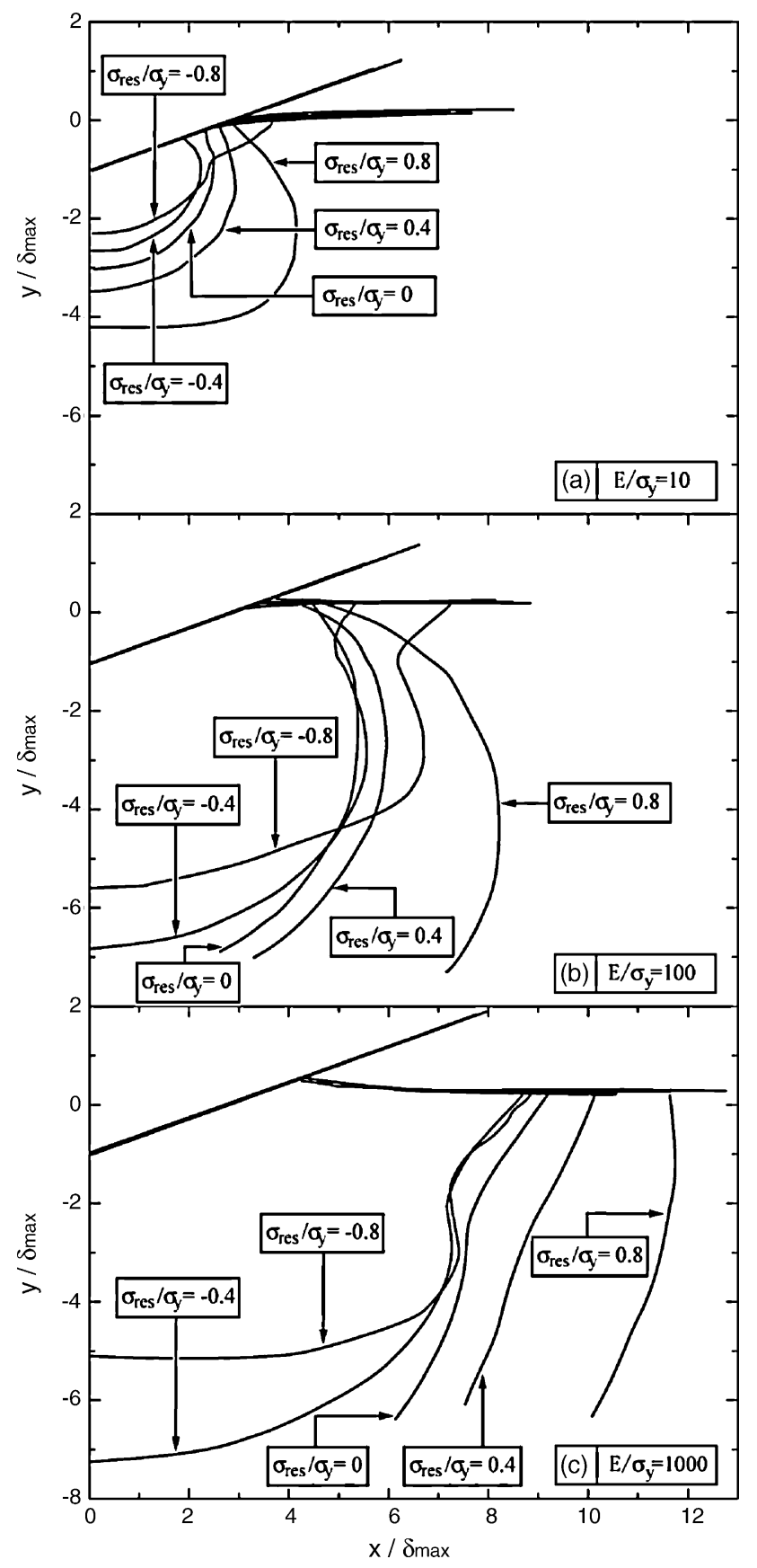

Fig. 3. Plastic zone as a function of residual stress showing the increase of plastic deformation with residual tension: (a) $E / \sigma_{\mathrm{y}}=10$; (b) $E / \sigma_{\mathrm{y}}=100$; (c) $E / \sigma_{\mathrm{y}}=1000$.

zone is very large beneath the indenter, hence, the elastic recovery is almost negligible.

The pile-up/sink-in amount $\left(\delta_{\mathrm{p}} / \delta\right)$ is thus dependent on both relative softness $\left(E / \sigma_{\mathrm{y}}\right.$, in consistent with literature $\left.[5,9,11]\right)$ and normalized residual stress $\left(\sigma_{\text {res }} / \sigma_{\mathrm{y}}\right.$, similar to the trend found in $[21,22])$. However, caution must be taken since the large difference between the surface profiles before and after unloading may lead to incorrect measurements of $\delta_{\mathrm{p}} / \delta$, as shown in Fig. 4a. As discussed in Section 2, it is therefore more reliable to represent the indentation work in the $2 \mathrm{D}$ space of $\left(E / \sigma_{\mathrm{y}}, \sigma_{\text {res }} / \sigma_{\mathrm{y}}\right)$, numerated below. 

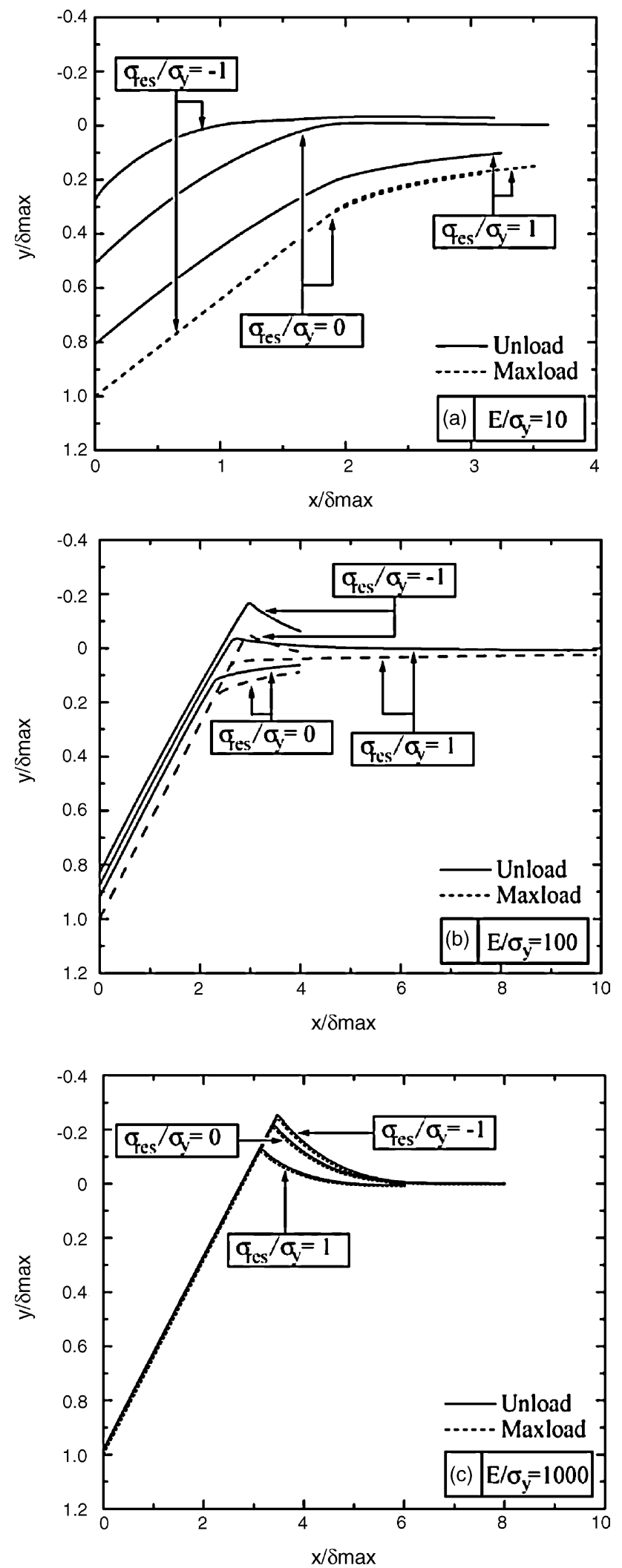

Fig. 4. The variation of surface profile near the indentation crater as a function of residual stress. Impression geometries for both maximum penetration and unloading are shown: (a) $E / \sigma_{\mathrm{y}}=10$; (b) $E / \sigma_{\mathrm{y}}=100$; (c) $E / \sigma_{\mathrm{y}}=1000$.

\subsection{Functional forms determined from indentation testing}

The functional forms of $f, g$, and $h$ (with reference to Eqs. (6), (7), and (11)) are shown in Fig. 5a-c, respectively. The normalized hardness is essentially a constant (about 2.9) when $E / \sigma_{\mathrm{y}}>300$, and the normalized stiffness is invariant (about 1.1) when $E / \sigma_{\mathrm{y}}>30$. This suggests that for soft materials, due to the finite plastic deformation induced by indentation, both hardness and stiffness are essentially insensitive to the residual stress. Consequently, they cannot be used to measure the residual stress in the substrate unless new equations (e.g. the indentation work) are taken into account. Tsui et al. [15] have found that for a soft aluminum alloy, both hardness and stiffness determined with the optically measured true contact area are essentially independent of the in-plane residual stress. This is in consistent with the current approach. Both limits of rigid plastic deformation, $c_{\mathrm{b}}=2.9$ (Fig. 5a) and $\gamma=1.1$ (Fig. 5b), agree with the values found in literature $[7,9,13]$.

When the substrate is not too soft $\left(E / \sigma_{\mathrm{y}}<300\right.$, which is applicable to many high-strength alloys and ceramic coatings), the existence of a tensile in-plane residual stress significantly enhances the plastic flow in the specimen, which produces a larger hardness impression and a lower measured hardness (Fig. 5a). The normalized hardness also decreases with increasing yield strain of the substrate, thanks to the increasing importance of elasticity. In absent of residual stress, the dependence of normalized hardness versus yield strain agrees well with the classic analysis by Johnson [9].

The residual stress significantly affects the contact stiffness when $E / \sigma_{\mathrm{y}}<30$. It is found from Fig. $5 \mathrm{~b}$ that tensile residual stress tends to increase the contact stiffness and residual compression will decrease the contact stiffness. Within a hard substrate, the presence of residual compression or tension will facilitate or impede the unloading process, and such effect amplifies with increasing elastic component $E / \sigma_{\mathrm{y}}$. The analysis of the unloading curves shows that more elastic work is recovered (both $S$ and residual indentation depth $\delta_{\mathrm{f}} / \delta_{\max }$ are smaller) with decreasing $E / \sigma_{\mathrm{y}}$ and decreasing $\sigma_{\text {res }} / \sigma_{\mathrm{y}}$, in consistent with Fig. 4. The normalized contact stiffness remains at constant $\gamma$ in absent of residual stress. Overall, Fig. 5a and $b$ suggests that both hardness and stiffness are sensitive to residual stress for high-strength materials with large yield strain.

The normalized indentation work (Fig. $5 \mathrm{c}$ ) appears to have a wide spread for both $E / \sigma_{\mathrm{y}}$ and $\sigma_{\text {res }} / \sigma_{\mathrm{y}}$. This is partly because the pile-up $\delta_{\mathrm{p}} / \delta$ varies with both yield strain and residual stress (c.f. Fig. 4). In general, the normalized indentation work increases with increasing $E / \sigma_{\mathrm{y}}$ : that is, in order to create the same impression, larger load (or plastic work) is needed to indent the substrate with higher modulus. Since the residual tension "facilitates" the penetration of indenter tip, the normalized indentation work decreases with increasing $\sigma_{\text {res }} / \sigma_{\mathrm{y}}$.

The results presented in the previous section indicate that the indentation testing is highly dependent on residual stresses, in particular for materials with $E / \sigma_{\mathrm{y}}<300$ and thus have important applications for high-strength materials. Fig. 5 suggests that when combined together, the normalized hardness, stiffness and 

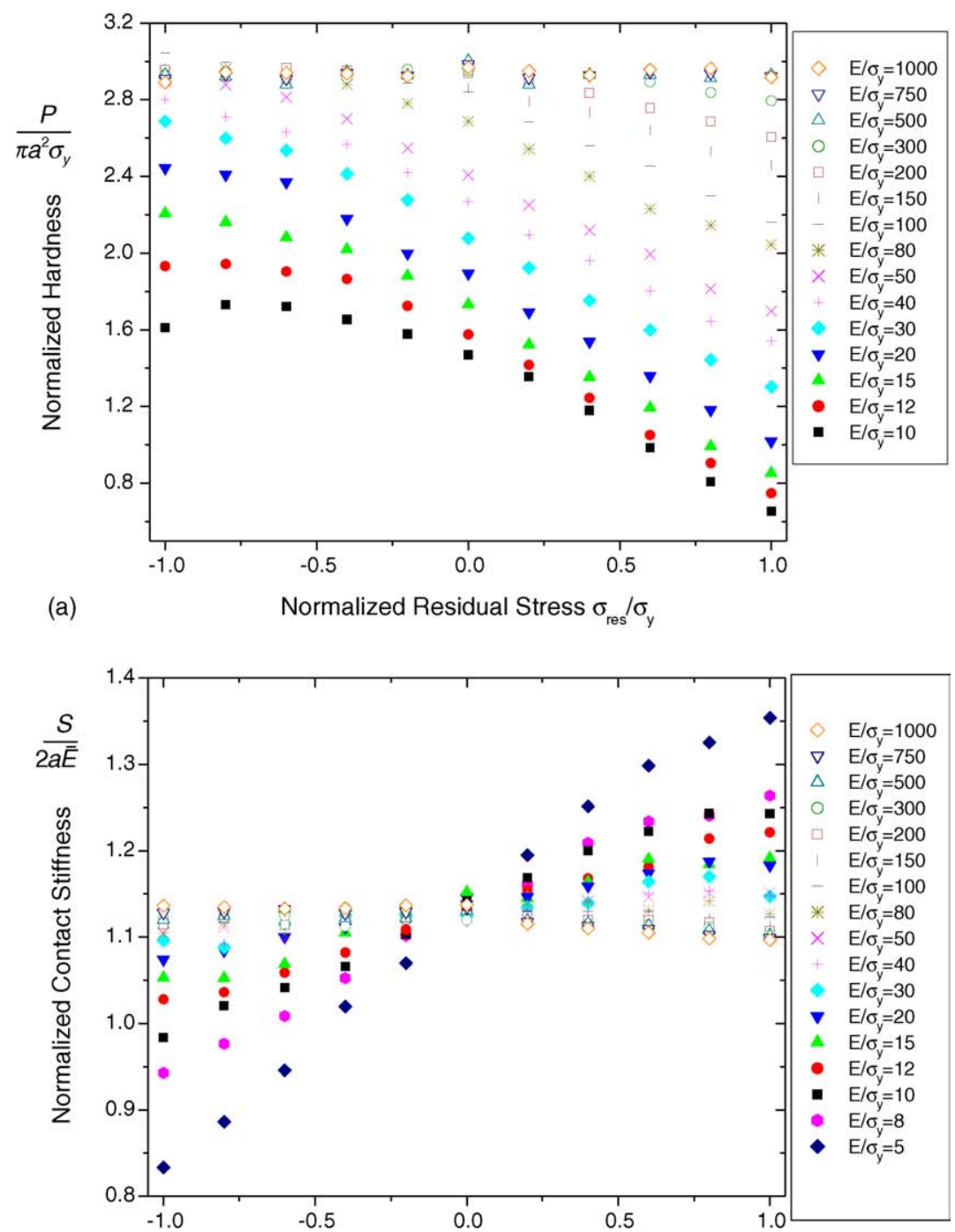

(b)

Normalized Residual Stress $\sigma_{\text {res }} / \sigma_{\mathrm{y}}$

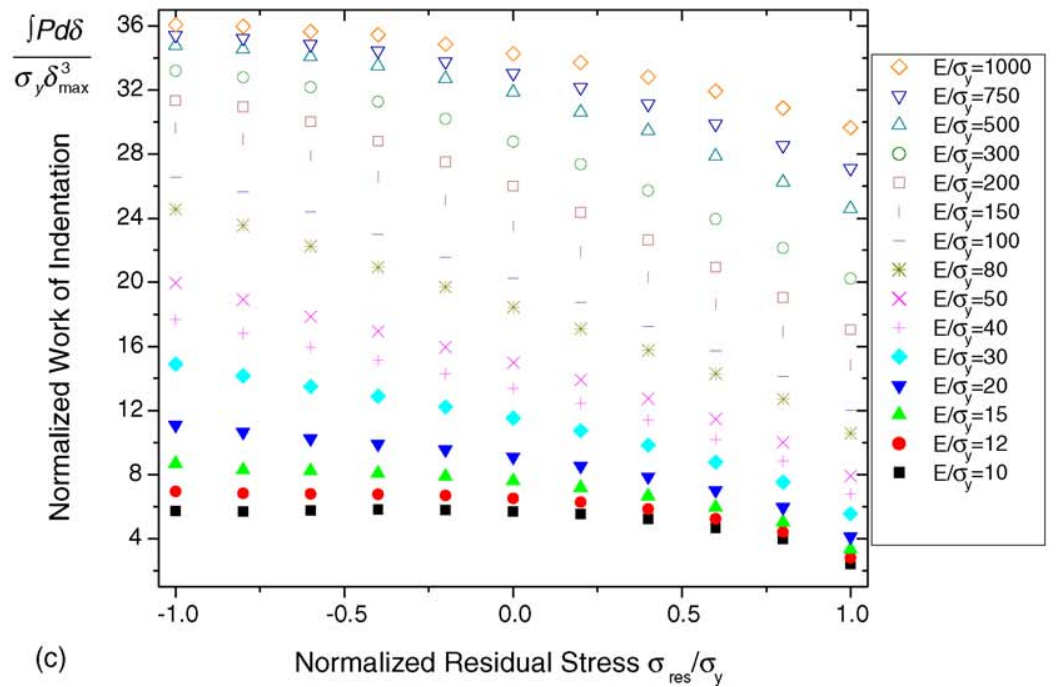

Fig. 5. (a) Normalized hardness $P / \pi^{2} \sigma_{\mathrm{y}}$, (b) normalized contact stiffness $S / 2 a \bar{E}$, and (c) normalized indentation work ${ }_{0}^{\delta_{\max }} P d \delta / \delta_{\max }^{3} \sigma_{\mathrm{y}}$, as a function of material elastic-plastic property $E / \sigma_{\mathrm{y}}$ and normalized yield stress $\sigma_{\text {res }} / \sigma_{\mathrm{y}}$. 

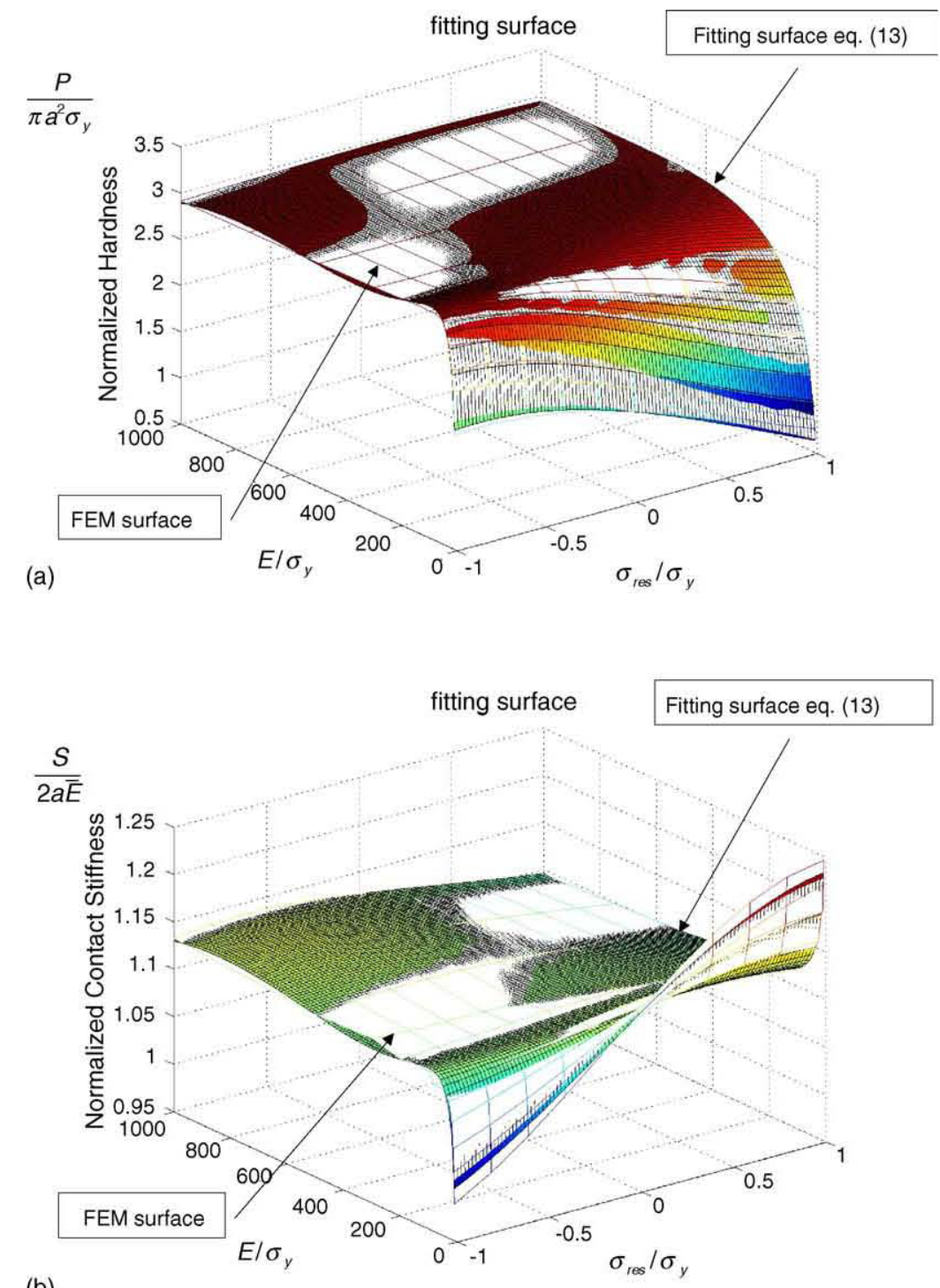

(b)

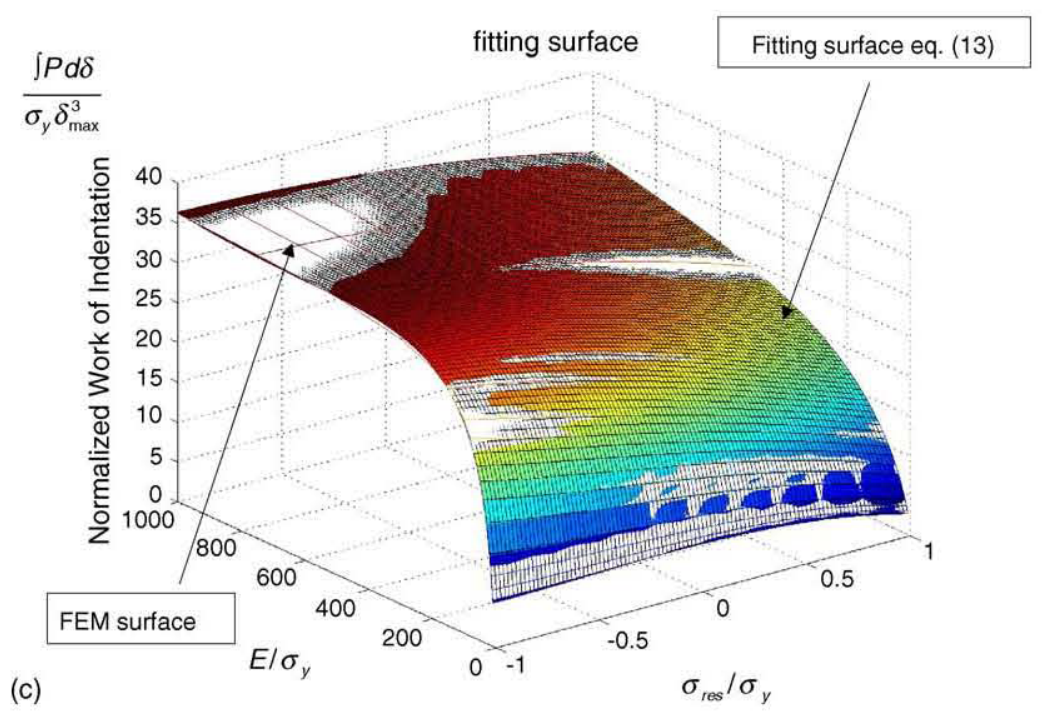

Fig. 6. Comparison between the finite element results (Fig. 5) and fitting function (13): (a) normalized hardness, (b) normalized contact stiffness, and (c) normalized indentation work. 
indentation work plots should yield sufficient information for the reverse analysis, from which the three unknowns $\left(E, \sigma_{\mathrm{y}}\right.$, $\sigma_{\text {res }}$ ) can be measured by indentation. Over the entire region of parameters considered in this study, the three functional forms $f, g$, and $h$ can be fitted to the following functions. The comparisons between fitted surfaces (Eq. (13)) with original data obtained from FEM (Fig. 5a-c) are shown in Fig. 6a-c. The fitting has an uncertainty less than $3 \%$ over the entire $\left(E / \sigma_{\mathrm{y}}\right.$, $\left.\sigma_{\text {res }} / \sigma_{\mathrm{y}}\right)$ space:

$$
\begin{aligned}
\Omega= & a_{1}+a_{2} \tau+a_{3} \tau^{2}+a_{4} \sqrt{\tau}+a_{5} \sqrt[3]{\tau}+a_{6} \xi+a_{7} \xi \tau \\
& +a_{8} \xi \tau^{2}+a_{9} \xi \sqrt{\tau}+a_{10} \xi \sqrt[3]{\tau}+a_{11} \xi^{2}+a_{12} \xi^{2} \tau \\
& +a_{13} \xi^{2} \tau^{2}+a_{14} \xi^{2} \sqrt{\tau}+a_{15} \xi^{2} \sqrt[3]{\tau}+a_{16} \xi^{3} \\
& +a_{17} \xi^{3} \tau+a_{18} \xi^{3} \tau^{2}+a_{19} \xi^{3} \sqrt{\tau} a_{20}+\xi^{3} \sqrt[3]{\tau}
\end{aligned}
$$

In Eq. (13), The function $\Omega$ represents normalized hardness $\left(P / \pi a^{2} \sigma_{\mathrm{y}}\right.$, or $\left.f\right)$, normalized contact stiffness $(S / 2 a \bar{E}$, or $g)$, or normalized indentation work $\left({ }_{0}^{\delta_{\max }} P d \delta / \delta_{\max }^{3} \sigma_{y}\right.$, or $\left.h\right)$, and $\tau \equiv E / \sigma_{\mathrm{y}}$ and $\xi \equiv \sigma_{\text {res }} / \sigma_{\mathrm{y}}$ are two variables. The coefficients $a_{i}(i=1-20)$ are listed in Table 1 .

\section{Reverse analysis and examples}

In order to solve the three unknowns $\left(E, \sigma_{\mathrm{y}}, \sigma_{\text {res }}\right)$ from the three fitting functions $(f, g, h)$, the optimization method is used to minimize the total error of Eq. (12). A flow chart of the reverse analysis based on such algorithm is given in Fig. 7. The hardness, contact stiffness, and indentation work are measured from an indentation load-displacement curve after the experiment. For each numerical iteration, the variables $\left(E, \sigma_{\mathrm{y}}, \sigma_{\text {res }}\right)$ are substituted in Eq. (13) and the error between $\Omega$ and the corresponding measured values are found. A total error function is defined as the summation of errors from $f, g$, and $h$. The roots $\left(E, \sigma_{\mathrm{y}}\right.$, $\left.\sigma_{\text {res }}\right)$ are found by searching a minimum of the total error function of those three variables on a fixed interval. The algorithm is based on golden section search and parabolic interpolation. The initial boundary are given by a rough range of the Young's

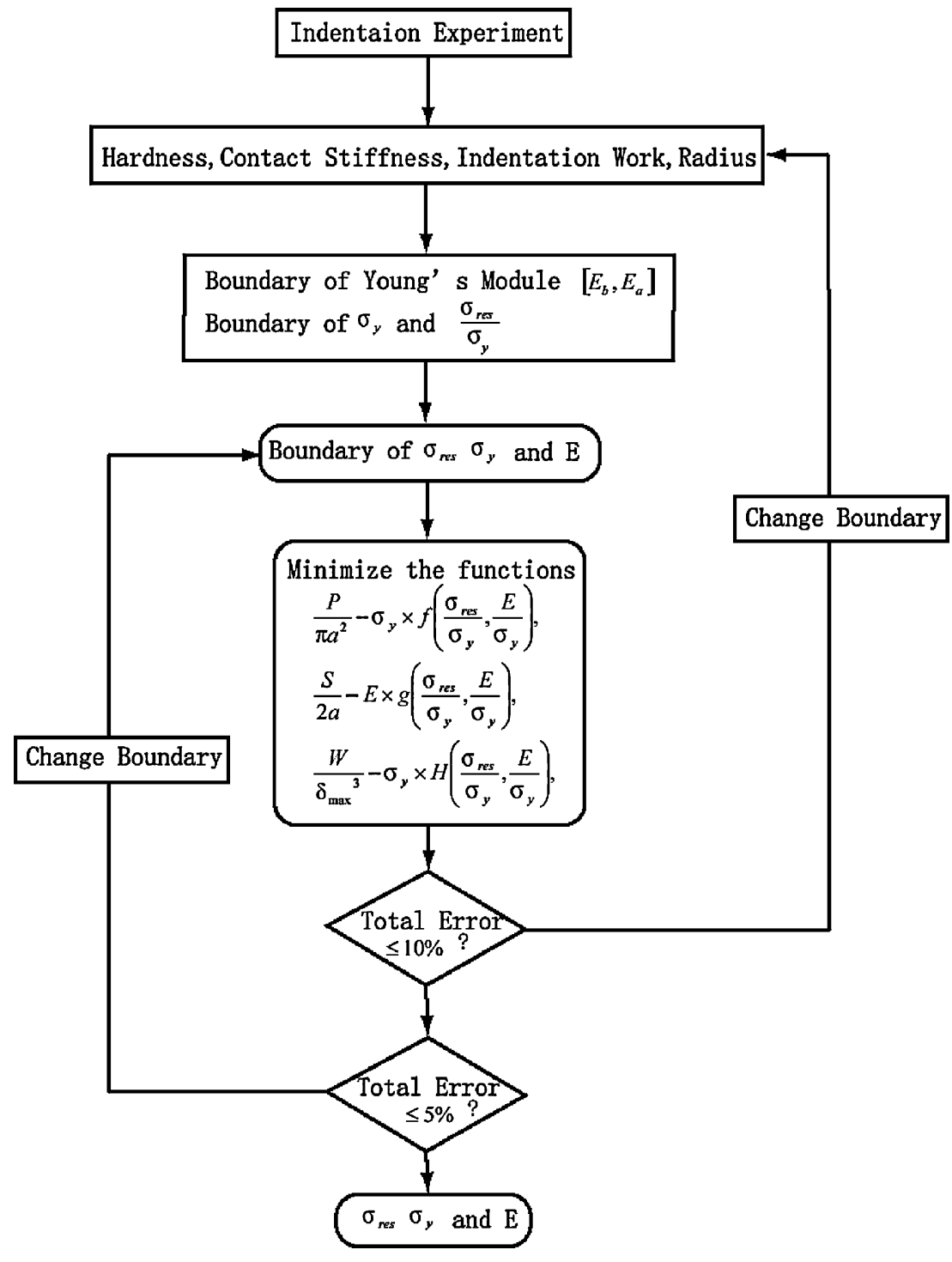

Fig. 7. Schematic of the process flow of reverse analysis. 
Table 1

Parameters of three fitting functional forms

\begin{tabular}{lccc}
\hline Parameters & $\begin{array}{l}\text { Normalized } \\
\text { hardness }\end{array}$ & $\begin{array}{l}\text { Normalized contact } \\
\text { stiffness }\end{array}$ & $\begin{array}{l}\text { Normalized work of } \\
\text { indentation }\end{array}$ \\
\hline$a_{1}$ & -1.4943 & 1.1041 & -3.7438 \\
$a_{2}$ & 0.0032 & $3.7528 \times 10^{-4}$ & -0.0771 \\
$a_{3}$ & $4.1178 \times 10^{-7}$ & $-8.5558 \times 10^{-8}$ & $1.7110 \times 10^{-5}$ \\
$a_{4}$ & -0.7500 & -0.0235 & 2.9685 \\
$a_{5}$ & 2.4572 & 0.0476 & 0.4208 \\
$a_{6}$ & 2.5012 & 0.8105 & 8.9619 \\
$a_{7}$ & -0.0244 & -0.0032 & 0.0507 \\
$a_{8}$ & $4.9842 \times 10^{-6}$ & $6.1966 \times 10^{-7}$ & $-1.9059 \times 10^{-5}$ \\
$a_{9}$ & 1.8116 & 0.2781 & 0.0963 \\
$a_{10}$ & -4.0314 & -0.7044 & -4.6897 \\
$a_{11}$ & -1.4904 & -0.0783 & -8.1284 \\
$a_{12}$ & 0.0095 & $2.8227 \times 10^{-4}$ & 0.0270 \\
$a_{13}$ & $-2.4728 \times 10^{-6}$ & $-5.2211 \times 10^{-8}$ & $-2.0045 \times 10^{-6}$ \\
$a_{14}$ & -0.6405 & -0.0258 & -2.7352 \\
$a_{15}$ & 1.4709 & 0.0649 & 6.8228 \\
$a_{16}$ & -1.0050 & -0.1904 & 5.9693 \\
$a_{17}$ & 0.0089 & 0.0011 & -0.0691 \\
$a_{18}$ & $-1.9143 \times 10^{-6}$ & $-2.3340 \times 10^{-7}$ & $1.6321 \times 10^{-5}$ \\
$a_{19}$ & -0.6334 & -0.0786 & 4.5106 \\
$a_{20}$ & 1.4098 & 0.1864 & -9.5823 \\
\hline & & & \\
\hline & & & \\
\hline
\end{tabular}

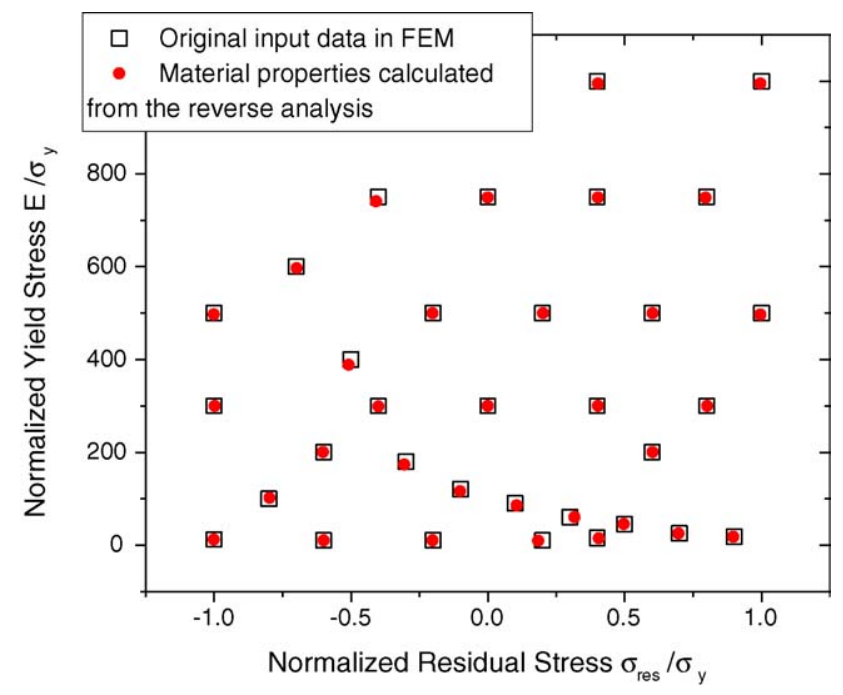

Fig. 8. Comparison between the material properties predicted from reverse analysis and the input parameters used in numerical indentation experiments.

modulus $E$, and an initial guess of both $\sigma_{\mathrm{y}} / E$ and $\sigma_{\text {res }} / \sigma_{\mathrm{y}}$. Based on the searching results obtained in the last numerical step, the boundary is updated automatically to give faster convergence and more accurate local solutions. An accurate solution with total error less than $5 \%$ can be obtained after several numerical iterations.

In order to examine the accuracy of reverse analysis, a total of 33 numerical experiments of indentation are performed, with $\sigma_{\mathrm{y}} / E$ and $\sigma_{\text {res }} / \sigma_{\mathrm{y}}$ varying in a large range. ${ }^{4}$ The input material parameters used in FEM are shown as open squares in Fig. 8. For each experiment, the contact hardness, stiffness, indentation

\footnotetext{
${ }^{4}$ Note that some of these parametric combinations where not used in generating Figs. 5 and 6.
}

work and projected contact area are measured from the finite element analysis. These numbers are then fed into the reverse analysis to predict $\sigma_{\mathrm{y}} / E$ and $\sigma_{\text {res }} / \sigma_{\mathrm{y}}$. The results obtained from numerical analysis are plotted as solid circles in Fig. 8. Excellent agreements between the original input data and reverse analysis are found for all possible combinations of residual stress and material parameters.

It would be ideal to compare the model with existing experimental data. Unfortunately, a complete set of experimental data which involves Berkovich indentation load-displacement curves for biaxially stressed elastic-perfectly plastic bulk material could not be found in literature. For example, Tsui et al. [15] and Suresh Giannakopoulos [17] did not show the variation of indentation load-displacement curves with residual stress (which is required for analyzing the indentation work in this study); while Swadener et al. [18] have used spherical indenters. Some materials that have been used in experiments have either significant strain hardening [20] (for low-carbon steel) or strain gradient effect at small indentation depth [19] (for single crystal tungsten), which leads to a normalized hardness far above 3 and thus does not apply to the present investigation. Some others only involve uniaxial residual stress instead of biaxial stress [22,25], or excessive substrate effect for indentation on biaxially stressed thin films [26]. Therefore, although bulk materials and coatings with equi-biaxial residual stress are very common in applications, to our knowledge, the existing indentation data in literature does not yield sufficient information to compare with the present model. Indentation experiments that include desired parameter outputs are currently in progress, and will be presented at a later date.

\section{Conclusion}

In this study, the finite element analysis has been used to investigate the effect of in-plane equi-biaxial residual stress on hardness and stiffness measured from indentation tests on a bulk material (or thick coating). By varying the material parameter and residual stress over a large range, it is found that both hardness and stiffness vary with residual stress, especially for high-strength materials with large yield strain. Both the plastic pile-up (or elastic sink-in) and indentation work are found to be very sensitive to the level of residual stress, which makes the determination of residual stress from indentation test possible. Based on the functional forms of normalized contact hardness, stiffness, and indentation work, a reverse algorithm is presented which may be used to measure the material yield stress, modulus, and residual stress from one simple indentation test. The comparison between the material properties predicted from reverse analysis and the input parameters used in numerical indentation experiments shows good agreement.

The effects of residual stress on indentation stress and plastic zone are also investigated. It is found that the indentation compressive stress diminishes with residual tension, which leads to an enlarged plastic zone and gives apparent lower hardness. On the other hand, with residual compression the maximum indentation compressive stress is increased, causing less plastic deformation (with smaller plastic zone) and gives rise to the 
hardness. It is also found that the elastic recovery upon unloading is very large for materials with high yield strain, and the elastic recovery increases with increasing residual compression. Therefore, caution must be taken when measuring the contact depth after unloading.

Unlike many of the previous studies [17-20], the new indentation technique proposed in this paper does not require a reference stress-free material for comparison purposes. Thus, this method has the potential to map the residual stress field on the surface of a specimen quickly and effectively.

\section{Acknowledgements}

The work of XC is supported in part by NSF CMS-0407743, and in part by the Department of Civil Engineering and Engineering Mechanics, Columbia University. The work of JY and AMK is supported by NSF DMR-0346664 and ONR N0001404-1-0498.

\section{References}

[1] J.W. Hutchinson, Z. Suo, Adv. Appl. Mech. 29 (1992) 63-191.

[2] V.K. Tolpygo, D.R. Clarke, K.S. Murphy, Surf. Coatings Technol. 146 (2001) 124-131.

[3] V. Teixeira, M. Andritschky, W. Fischer, H.P. Buchkremer, D. Stover, J. Mater. Process. Technol. 92 (1999) 209-216.
[4] J. Shi, S. Darzens, A.M. Karlsson, Mater. Sci. Eng. A 392 (2005) 301-312.

[5] W.C. Oliver, G.M. Pharr, J. Mater. Res. 7 (1992) 1564-1583.

[6] F. Doerner, W.D. Nix, J. Mater. Res. 1 (1986) 601-609.

[7] G.M. Pharr, Mater. Sci. Eng. A 253 (1998) 151-159.

[8] G.M. Pharr, A. Bolshakov, J. Mater. Res. 17 (2002) 2660-2671.

[9] K.L. Johnson, Contact Mechanics, Cambridge University Press, 1985.

[10] J.J. Vlassak, W.D. Nix, J. Mech. Phys. Solids 42 (1994) 1223-1245.

[11] X. Chen, J.J. Vlassak, J. Mater. Res. 16 (2001) 2974-2982.

[12] R.B. King, Int. J. Solids Struct. 23 (1987) 1657-1664.

[13] J.C. Hay, A. Bolshakov, G.M. Pharr, J. Mater. Res. 14 (1999) 2296-2305.

[14] N.A. Fleck, J.W. Hutchinson, Adv. Appl. Mech. 33 (1997) 295-361.

[15] T.Y. Tsui, W.C. Oliver, G.M. Pharr, J. Mater. Res. 11 (1996) 752 759 .

[16] A. Bolshakov, W.C. Oliver, G.M. Pharr, J. Mater. Res. 11 (1996) $760-768$.

[17] S. Suresh, A.E. Giannakopoulos, Acta Mater. 46 (1998) 5755-5767.

[18] J.G. Swadener, B. Taljat, G.M. Pharr, J. Mater. Res. 16 (2001) 2091-2102.

[19] Y.H. Lee, D. Kwon, Scripta Mater. 49 (2003) 459-465.

[20] Y.H. Lee, D. Kwon, Acta Mater. 52 (2004) 1555-1563.

[21] S. Carlsson, P.L. Larsson, Acta Mater. 49 (2001) 2179-2191.

[22] S. Carlsson, P.L. Larsson, Acta Mater. 49 (2001) 2193-2203.

[23] S.D. Mesarovic, N.A. Fleck, Proc. Roy. Soc. London A455 (1999) 2707-2728.

[24] ABAQUS, ABAQUS 5.8 User's Manual, ABAQUS Inc., 1998.

[25] A.V. Zagrebelny, C.B. Carter, Scripta Mater. 37 (1997) 1869-1875.

[26] G. Wei, B. Bhushan, S.J. Jacobs, Ultramicroscopy 100 (2004) 375389. 